Die Bestimmung von Mikrogramm-Mengen von Platinmetallen in Lösungen. V.A. Orobinskaja und N.E. Čulkova.

Best. von Platinmetallen in Lösungen; Chromatographie, Säulen; modif. Cellulosefaser.

Es werden Ergebnisse von Untersuchungen zur Konzentrierung von Platinmetallen aus komplexen Gemischen an einer Cellulosefaser, die modifiziert worden war und Thioamidgruppen enthielt (Mtilon-T) dargelegt. Die Untersuchungen wurden $z$. T. mit radioaktiven Platinmetallen durchgeführt, wobei besonders die Faktoren untersucht wurden, die die Sorption der Platinmetalle an der Faser beeinflussen, wie Acidität, Kontaktzeit, Konzentration u.a. Es wird eine Methode zur Bestimmung von Mikrogramm-Mengen an Platinmetallen in armen Lösungen vorgeschlagen.

Trudy 8. Sov. Chim. Anal. Techn. Blag. Met. 8, 90-95 (1971)(Russisch)

$$
\text { TIB }
$$

\section{Organische Verbindungen}

The Determination of the Nitrogen Content and Nitrogen-15 Abundance by Means of the Nitrogen Gas Generated from Inorganic and Organic Materials. R. Fiedler and G. Proksch.

Best. von Stickstoff und Stickstoff-15; Massenspektrometrie; nach Dumas-Verbrennung.

Für die Bestimmung des Gesamtstickstoffgehalts in verschiedenen Arten von Proben wird eine Methode beschrieben, die auf der Umwandlung zu Stickstoffgas beruht und sich für die anschließende massenspektrometrische Bestimmung der ${ }^{15} \mathrm{~N}$-Häufigkeit eignet. Die Probe wird mit einem Gemisch von Kupferoxid, Kupfer und Calciumoxid in einem geschlos senen Rohr unter Vakuum verbrannt (modifizierte Dumas-Methode). Das Gas wird in eine evakuierte Kammer mit bekanntem Volumen gelassen und aus dem Stickstoffdruck der Stickstoffgehalt der Probe ermittelt. Dann wird das Gas in ein Massenspektrometer überführt. Verschiedene Verbrennungstemperaturen, Verbrennungszeiten und Oxidationsmittel wurden in Hinblick auf vollständige Verbrennung des Materials und Bildung von reinem Stickstoff verglichen.

Anal. Chim. Acta 60, 277-285 (1972). I.A. E.A. Lab., Seibersdorf (Österreich) K. Henning

Automation in der organischen Analyse. I. Mikround Ultramikrobestimmung von Sauerstoff. K. Ubik, J.Horáček und V.Pechanec.

Best. von Sauerstoff in Organ. Verbindungen; Elementaranalyse; Mikro- und Ultramikrobest., automat.

Bei dem beschriebenen Verfahren zur Sauerstoffbestimmung in organischen Substanzen handelt es sich um die erste Methode, die die Verwendung eines aus platiniertem Ruß bestehenden Katalysators mit $\mathrm{H}_{2}$ als Trägergas und mit Wärmeleitfähigkeitsdetektion des zu bestimmenden Produktes verbindet. Es wurden einige bei der Pyrolyse sauerstoffhaltiger Substanzen verlaufenden Reaktionen wie die Reaktion von $\mathrm{H}_{2}$ an platiniertem $\mathrm{Ru} \beta$ und reinem Ruß, in Abhängigkeit von der Temperatur und der Schichtdicke des Kontaktes, die Reaktion von $\mathrm{H}_{2}$ mit der durch Pyrolyse entstandenen Substanz wie die Temperaturabhängigkeit der Methanzersetzung an platiniertem und reinem Ruß und die Temperaturabhängigkeit der Konversion von $\mathrm{CO}_{2}$ zu $\mathrm{CO}$ an platiniertem Ruß, die $\mathrm{Ab}-$ hängigkeit der $\mathrm{CO}-\mathrm{Beständigkeit} \mathrm{an} \mathrm{platiniertem} \mathrm{Ruß}$ im $\mathrm{H}_{2}$-Strom von der Menge zugeführten $\mathrm{CO}$ untersucht. Die Entfernung störender Bestandteile aus Verbindungen, die noch $\mathrm{S}$ und Halogene enthalten, gelingt,wenn ein Absorptionsröhrchen mit Natronasbest und ein $5 \&$ Molekularsieb zwischen Reaktionsrohr und Katharometer eingeschaltet wird. Basierend auf den Ergebnissen der Voruntersuchungen wurde eine Schnellanalysenmethode ausgearbeitet, deren Prinzip darauf beruht, daß nach der Pyrolyse der zu analysierenden Verbindungen im $\mathrm{H}_{2}$ Strom $\left(30 \mathrm{ml} / \mathrm{min}\right.$ ) bei $1000-1100^{\circ} \mathrm{C}$ und der Führung der Pyrolysenproduktion durch eine $8 \mathrm{~cm}$ dicke Schicht auf $1050^{\circ} \mathrm{C}$ erhitztem platiniertem Ruß $(50 \%$ Pt) und Absorptionsröhrchen, die Wärmeleitfähigkeit des Gasgemisches durch einen ZweikammernKatharometer bestimmt wird. Die Widerstandsmessung der Wheatstone'schen Brücke geht auf einen Schreiber, die Fläche der aufgezeichneten Kurve wird durch einen Integrator berechnet, der die Impulszahl ausdruckt. Eine Eichkurve (Impulszahl gegen $\mu \mathrm{g}$ O) wurde mit Standardsubstanz (Benzoesäure, Benzoin und n-Oktadekanol) aufgestellt. Eine Bestimmung dauert nur $12 \mathrm{~min}$, der absolute Fehler übersteigt nicht $\pm 0,3 \%$.

Coll. Czech. Chem. Commun. 37, 102-114 (1972). Inst. Org. Chem. Biochem, , Tschech. Akad. Wiss. Prag (Tschechoslowakei) G. Böck

Simultane Bestimmung von Schwefel und Chlor in organischen Verbindungen. I. V.Avguševič, E.S. Kulikova, A.A. Zacharova und J. M. Veličko.

Best. von Chlor und Schwefel in Organ. Verbindungen; Elementaranalyse.

Die Probe wird in Sauerstoff verbrannt, und die Verbrennungsprodukte werden in neutralem Wasserstoffperoxid absorbiert. In der Absorptionslösung werden Chloride durch mercurimetrische Titration gegen Diphenylcarbazon ermittelt und anschließend Sulfate in einer $80 \%$ igen Acetonlösung mit Bariumnitrat gegen Chlorphosphonazo als Indicator titriert. - Arbeitsweise. $3-10 \mathrm{mg}$ Probe werden auf die übliche$\bar{W}$ eise in einem Sauerstoffstrom von $20-25 \mathrm{ml} / \mathrm{min}$ verbrannt, die Verbrennungsprodukte werden in $3 \mathrm{ml}$ einer 6 - 7\%igen Wasserstoffperoxidlösung in einem mit GlaskugeIn gefüllten Absorber absorbiert. Hinter dem Absorber wird noch ein Kontrollkolben mit $7 \mathrm{ml}$ derselben Absorptionslösung eingeschaltet. Nach der beendeten Verbrennung wird die Lösung 
aus dem Absorber in den Kontrollkolben überführt, das Absorptionsgefäß und das Gasableitungsrohr werden mit dest. Wasser gespült und die Lösung wird auf $5 \mathrm{ml}$ abgedampft. $0,5 \mathrm{ml} 0,5 \mathrm{~N}$ Salpetersäure und $2-3 \mathrm{Tr}$. $1 \%$ ige äthanolische Diphenylcarbazonlösung werden zugesetzt und Chloride werden mit $0,01 \mathrm{~N}$ Quecksilber(II)nitrat bis zum Farbumschlag von Gelb nach Violett titriert. Dann wird durch Zusatz von einigen Tropfen Salzsäure der pH-Wert auf 1 3 eingestellt und gleichzeitig auch die violette Färbung des Indicators beseitigt. Ein vierfaches Volumen von Aceton und $2-3 \mathrm{Tr}$. 0,2\%ige äthanolische oder wäßrige Chlorphosphonazolösung werden zugesetzt und die Lösung wird mit einer 0, $2 \mathrm{~N}$ Bariumnitratlösung bis zum Farbumschlag von Violett nach Blau titriert. Chloride und Nitrate in einem 150 fachen Überschuß stören die Sulfatbestimmung nicht. Noch $50 \mu \mathrm{g} \mathrm{Cl}^{-}$und $30 \mu \mathrm{g} \mathrm{SO}_{4}^{2}$ - lassen sich bestimmen; das entspricht bei einer Einwage von $10 \mathrm{mg}$ Probe etwa $0,5 \%$ Chlor und 0,1\% Schwefel. Der Bestimmungsfehler ist nicht größer als 0,3\%. Die Analyse dauert $50-60 \mathrm{~min}$.

Zavodsk. Lab. 38, 150-152 (1972)(Russisch). Inst. f. fossile Brennstoffe, Moskau (UdSSR) M. Přibyl

Reaktionen des Dicyclopentadienyldiphenyltitans mit den Phenylderivaten von Lithium, Zink, Cadmium und Quecksilber. G. A. Razuvaev, V. N. Latjaeva, L. I. Vyšinskaja, T.P. Denisova und Ju. P. Gorelov.

Komplexbildung von Dicyclopentadienyldiphenyltitan mit Metallphenylderivaten, Phenylderivaten.

Dicyclopentadienyldiphenyltitan bildet bei $-40^{\circ} \mathrm{C}$ in Diäthyläther mit Phenyllithium den stabilen Komplex $\left[\left(\mathrm{C}_{5} \mathrm{H}_{5}\right)_{2} \quad \mathrm{Ti}\left(\mathrm{C}_{6} \mathrm{H}_{5}\right)_{3}\right]$ Li. Dieser gibt mit $\mathrm{MCl}_{2}(\mathrm{M}=$ $\mathrm{Zn}, \mathrm{Hg})\left[\left(\mathrm{C}_{5} \mathrm{H}_{5}\right)_{2} \mathrm{Ti}\left(\mathrm{C}_{6} \mathrm{H}_{5}\right)_{3}\right] 2^{\mathrm{M}}$. Die erwähnten Komplexe von $L i$ und $M$ sind bei Raumtemperatur unbeständig, und mit Methanol werden sie in $\left(\mathrm{C}_{5} \mathrm{H}_{5}\right)_{2} \mathrm{Ti}\left(\mathrm{C}_{6} \mathrm{H}_{5}\right)_{2}$, $\mathrm{C}_{6} \mathrm{H}_{6}$ und $\mathrm{LiOCH}_{3}$ oder $\mathrm{M}\left(\mathrm{OCH}_{3}\right)_{2}$ umgesetzt. $\left(\mathrm{C}_{5} \mathrm{H}_{5}\right)_{2} \mathrm{Ti}\left(\mathrm{C}_{6} \mathrm{H}_{5}\right)_{2}$ gibt mit $\left(\mathrm{C}_{6} \mathrm{H}_{5}\right)_{2} \mathrm{Zn}$ den Komplex $\left[\left(\mathrm{C}_{5} \mathrm{H}_{5}\right)_{2} \mathrm{Ti}\left(\mathrm{C}_{6} \mathrm{H}_{5}\right)\right]_{2} \mathrm{Zn}$, das dunkelrote Kristalle von Fp $217^{\circ} \mathrm{C}$ (mit Zersetzung) bildet, die thermisch beständig und in organischen Lösungsmitteln schwer lös lich sind. Die genannte Reaktion verläuft nach $30-40 \mathrm{~h}$ bei $60^{\circ} \mathrm{C}$ in ätherischer Lösung in geschlossenen Ampullen. Bei der Reaktion von $\left(\mathrm{C}_{5} \mathrm{H}_{5}\right)_{2} \mathrm{Ti}\left(\mathrm{C}_{6} \mathrm{H}_{5}\right)_{2}$ mit $\left(\mathrm{C}_{6} \mathrm{H}_{5}\right)_{2} \mathrm{Hg}$ oder $\left(\mathrm{C}_{6} \mathrm{H}_{5}\right)_{2} \mathrm{Cd}$ wird kein Komplex wie im Falle von $\mathrm{Zn}$ gebildet.

Dokl. Akad. Nauk SSSR 202, 1090-1092 (1972)(Russisch) Forsch. Inst. Chem., Staatl. Univ. Lobačevski, Gorki (UdSSR)

$$
\text { E. Svátek }
$$

Ammonium Hexanitratocerate(IV) as an Oxidizing Agent. VII. Determination of some Carboxylic Acids. G. G. Rao, I. Subrahmanyam and B. M. Rao.

Best. von Carbonsäuren; Volumetrie; Oxidation mit Ammoniumhexanitratocerat(IV).
Ammoniumhexanitratocerat(IV); als oxidierendes Reagens.

Einige Carbonsäuren werden mit Ammoniumhexanitratcerat(IV) stöchiometrisch oxidiert. Als Endprodukt entsteht Ameisensäure, die dadurch selbst nicht bestimmbar ist. Zur Bestimmung gibt man zur Probe $5 \mathrm{ml} 10 \mathrm{M}$ Salpetersäure, verdünnt auf $100 \mathrm{ml}$ und erhitzt $30 \mathrm{~min}$ lang auf $60^{\circ} \mathrm{C}$. Nach dem Kühlen gibt man $20 \mathrm{ml} 5 \mathrm{~N}$ Schwefelsäure hinzu und titriert den Überschuß an Cer(IV) mit Eisen(II) gegen Ferroin zurück. Erforderlich sind 6, 14 bzw. 8 Mol Cer(IV) für Wein- und Malonsäure, Citronensäure bzw. Äpfelsäure.

Talanta 19, 1083 (1972). Dept. Chem., Andhra Univ., Waltair (Indien)

$$
\mathrm{K} \text {. Henning }
$$

Glyoxalin als neues Titrationsmittel für die Mikrobestimmung von Fumarsäure. A. K. Saxena.

Best. von Fumarsäure mit Glyoxalin; Volumetrie; Mikromaßstab.

Verf. berichtet über die Mikrobestimmung von Fumarsäure mit Glyoxalin und Bromkresolpurpur als Indikator, wobei $0,058-0,58 \mathrm{mg}$ mit einem maximalen Fehler von $\pm 0,004 \mathrm{mg}$ nachgewiesen werden konnten. - Ausführung: eine $15 \mathrm{ml}$-Probe versetzt man mit $1=2$ Tr. einer 0, $1 \%$ igen Bromkresolpurpurlösung und titriert die gelbgefärbte Lösung mit dem Reagens nach Schwachpurpurfarben.

Chim. Anal. (Paris) 53, 567--568 (1971). Dept. Chem., Univ. Allahabad (Indien) L. Johannsen

Die Verwendung von Kaliumruthenat als Katalysator in der Titrimetrie. P.K. Norkus und Ju. Ju. Jankauskas.

Best. von Weinsäure mit Perjodat und Kaliumruthenat; Volumetrie; $\mathrm{K}_{2} \mathrm{RuO}_{4}$ als Katalysator.

In dieser Arbeit werden die Bedingungen zur Bestimmung von Weinsäure und deren Salzen mit Perjodat unter Anwendung eines Katalysators - $\mathrm{K}_{2} \mathrm{RuO}_{4}$ - beschrieben. - Zur Analysenlösung werden $>2 \mathrm{ml} 0,1 \mathrm{~N}-$ Lösung von $\mathrm{K}-$ Perjodat zugegeben und auf $\mathrm{pH} 3-6$. eingestellt. Innerhalb der nächsten $1-2$ min wird die Lösung auf $0,2-1 \mathrm{~N} \mathrm{OH}^{-}$-Konzentration gebracht, 3 - $4 \mathrm{Tr}$. 0, 2\%ige $\mathrm{K}_{2} \mathrm{RuO}_{4}$-Lösung zugegeben, das Potential mit einer Platinelektrode innerhalb 1 - 2 min bis $150-200 \mathrm{mV}$ verfolgt und der Überschuß des

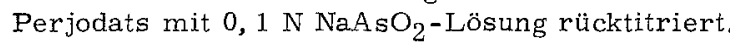
Bei der Berechnung wird davon ausgegangen, da $\beta$ 3 Mol Perjodat einem Mol Weinsäure oder Tartrat entsprechen. Bei der Analyse von Ammoniumtartrat wurde die 3-fache Katalysatormenge zugegeben (das Ammonion desaktiviert z. T. den Katalysator). Das in manchen Lösungen störende Kupfer wurde mittels Ionenaustauscher entfernt.

Ž. Anal. Chim. 26, 2051-2053 (1971)(Russisch, mit engI. Zus.fass.). Inst. Chemie u. Chem. Technologie, Akad. Wiss, der Lit. SSR, Wilna (UdSSR)

$$
\text { P. Dolezel }
$$


Total Carbohydrate Determination in Dimethyl Sulfoxide and Guanidin Hydrochloride as Solvents. F.R. Dintzis.

Best, von Kohlenhydraten; Autoanalysator; neue Lösungsmittel für die Phenol- $\mathrm{H}_{2} \mathrm{SO}_{4}-$ Methode.

Zur Bestimmung der Gesamtkohlenhydrate mit der Phenol-Schwefelsäuremethode von M. Duboir, K. A. Giles, J.K. Hamilton, P.A. Rebers und F. Smith (Anal. Chem. 28, 350 (1956)) gibt Verf. zwei Verfahren für den AutoAnalyzer an. Bei dem einen dient Dimethylsulfoxid, bei dem anderen $4 \mathrm{M}$ Guanidinhydrochlorid als Lösungsmittel. Jedem Verfahren ist ein Fließdiagramm beigegeben.

Anal. Biochem. 46, 1C8-113 (1972). Northern Reg. Res. Lab., Peoria, Ill. (USA)

$$
\text { E. Müller, Marburg }
$$

Infrarotspektroskopische Analyse von durch IndenPseudonitrositen emittierten Gasen bei Erhöhung der Temperatur. M. Colette.

Analyse von Indenpseudonitrositen; Spektralphotometrie, IR; nach thermischer Zersetzung.

Die Indenpseudonitrosite haben je nach Temperatur einen verschiedenen Zersetzungsmechanismus. Bis $50^{\circ} \mathrm{C}$ entstehen hauptsächlich Stickstoffmonoxid und Distickstoffmonoxid. Ab $65^{\circ} \mathrm{C}$ werden hauptsächlich Kohlendioxid und Stickstoff als Zersetzungsgase beobachtet. Zur Analyse werden $100 \mathrm{mg}$ des Pseudonitrosits in eine Gaszelle gegeben. Die entstehenden Gase werden nach Erhitzung auf verschiedene Temperaturen IR-spektroskopisch analysiert. Die charakteristischen Banden für eine $\mathrm{NO} / \mathrm{N}_{2} \mathrm{O}$-Mischung liegen bei 1350,1600 und $1630 \mathrm{~cm}^{-1}$. NO wird durch das Dublett im Bereich von $1800 \mathrm{~cm}^{-1}$ charakterisiert. Banden bei 590 und $820 \mathrm{~cm}^{-1}$ werden zur $\mathrm{CO}_{2}$ Charakterisierung verwendet.

C. R. Acad. Sci. (Paris) Sér. C, 274, 1047-1050 (1972). Fac. Sci., Dept. Chim., Campus Universitaire, Tunis (Tunesien) R. H. Sterzel

Säure-Basen-Eigens chaften von Xanthenverbindungen. O. V. Sivanova, G. S. Ivankovič und G. S. Lagutina.

Untersuchung von Xanthenverbindungen, Phenylfluorene; Spektralphotometrie; Säure-Base-Eigenschaften.

In der Arbeit werden Phenylfluoron(I) und dessen o-, $\mathrm{m}$ - und $\mathrm{p}$-Nitroderivate(II, III und IV) eingehend spektralphotometrisch untersucht. Bei $20-25^{\circ} \mathrm{C}$ wird die optische Dichte im Bereich von 400 bis $700 \mathrm{~nm}$ in Abhängigkeit vom $\mathrm{pH}-$ Wert gemessen ( $\mathrm{pH}$ variiert zwischen 0,25 und 13,90, mit Salzsäure oder Kaliumhydroxid eingestellt). Die Arbeitslösungen enthalten je 25\% Alkohol, damit die Koagulation der Phenylfluorone ausgeschaltet wird. Bei $\mathrm{pH}-$ Werten unterhalb von 2 hängt die Lichtabsorption nicht von dex Acidität $a b$; dies ist durch das Vorhandensein der einwertigen Kationen $\mathrm{H}_{4} \mathrm{R}^{+}$bedingt, deren Lichtabsorptionsmaxima für die einzelnen Stoffe der Reihe nach bei $\lambda_{1}=$ 465; $\lambda_{2}=470 ; \lambda_{3}=470 ; \lambda_{4}=475 \mathrm{~nm}$ liegen. Nimmt der pH-Wert zu, nimmt die kurzwellige Lichtabsorp- tion ab, während sie bei längeren Wellen in dem Maße zunimmt, wie die neutralen Moleküle angehäuft werden, deren Lichtabsorptionsmaxima sich bei $490,495,495$ und 500 befinden. Die isosbestischen Punkte treten deutlich in Erscheinung bei $480,485,490$ und $490 \mathrm{~nm}$ und lassen das einfache Gleichgewicht zwischen den Kationen und den neutralen Molekülen erkennen. Die weitere Deprotonisierung ist durch einen bathochromen und hyperchromen Effekt gekennzeichnet. Die einwertigen Anionen $\mathrm{H}_{2} \mathrm{R}^{-}$ absorbieren am stärksten bei $515,525,525$ und $525 \mathrm{~nm}$. Die entsprechenden isosbestischen Punkte liegen bei 495, 495, 500 und $500 \mathrm{~nm}$. Die zweiwertigen Anionen absorbieren am stärksten bei denselben Wellenlängen wie die einwertigen, ihre Lichtabsorption ist jedoch schwächer. In stark alkalischem Milieu sind dreiwertige Anionen vorhanden, deren Lichtabsorptionsmaxima bei $560,560,565$ und $565 \mathrm{~nm}$ liegen; für die isosbestischen Punkte ergeben sich die Wellenlängen 535,540,540 und $555 \mathrm{~nm}$. Auf Grund der spektralphotometrischen Angaben sind die stufenweisen Dissoziationskonstanten der vier Stoffe ermittelt worden, und zwar mit Hilfe von zwei verschiedenen Methoden (graphisch, elektronenrechnerisch). Die Werte der Dissoziationskonstanten sind in einer Tabelle zusammengestellt. Beide Methoden ergeben Werte, die gut miteinander übereinstimmen. Ebenfalls ist die prozentuale Vertretung der einzelnen Ionenformen in Abhängigkeit vom $\mathrm{pH}$-Wert berechnet worden. Die Verteilung der Formen von p-Nitrophenylfluoron ist in einer Abbildung des Originals dargestellt. Die Nitrosubstitution bewirkt eine Zunahme des sauren Charakters.

Ž. Anal. Chim. 26, 1874-1879 (1971)(Russisch, mit engl. Zus. fass.). Cernyševskij Staatl. Univ., Saratov (UdSSR) B. Tvaroha

A Capillary Column für the Gas Chromatographic Analysis of Dicyclopentadiene. W.F. Tully.

Analyse von Dicyclopentadien; Chromatographie, Gas. Die Darstellung von Dicyclopentadien (DCPD) erfolgt durch Dimerisierung von Cyclopentadien nach DielsAlder. Für die analytische Bestimmung von DCPD, die durch begleitende Codimere von Cyclopentadien und u.a. Batadien sowie Isopren erschwert wird, ist die Gas-Flüssig-Chromatographie gut geeignet. -

Arbeits bedingungen. Perkin-Elmer-Gas-Chromatograph Modē 900 , Golay-Kolonne $4,5 \mathrm{~m} \times 0,8 \mathrm{~mm}$, Säulenfüllung $\mathrm{m}-\mathrm{Bis}(\mathrm{m}-\mathrm{phenoxy}$ phenoxy)benzol mit $2 \%$ Apiezon L (MBMA), Trägergas He, Temperatur $100-180^{\circ} \mathrm{C}\left(32^{\circ} / \mathrm{min}\right)$, FID.

J. Chromatog. Sci. 9, 635-638 (1971). Res. Develop. Dept., Union Carbide Corp., Chem. Plastics Div. South Charleston, W. Va. 25303 (USA)

K. Dornberger 